Cahiers d'études africaines

169-170|2003

Enseignements

\title{
École et mondialisation
}

Vers un nouvel ordre scolaire?

Marie-France Lange

\section{CpenEdition}

Journals

Édition électronique

URL : https://journals.openedition.org/etudesafricaines/194

DOI : 10.4000/etudesafricaines. 194

ISSN : $1777-5353$

Éditeur

Éditions de l'EHESS

Édition imprimée

Date de publication : 1 janvier 2003

Pagination : 143-166

ISBN : 978-2-7132-1809-5

ISSN : 0008-0055

Référence électronique

Marie-France Lange, «École et mondialisation », Cahiers d'études africaines [En ligne], 169-170 | 2003, mis en ligne le 20 décembre 2006, consulté le 21 septembre 2021. URL : http://

journals.openedition.org/etudesafricaines/194 ; DOI : https://doi.org/10.4000/etudesafricaines.194

(c) Cahiers d'Études africaines 


\section{Marie-France Lange}

\section{École et mondialisation Vers un nouvel ordre scolaire?}

La mondialisation est souvent perçue comme la mise en place d'un nouveau système de domination fondé sur l'imposition de la logique de marché à toutes les sphères des sociétés (économique, sociale, politique, culturelle, éducative...). Le débat théorique sur la mondialisation n'est guère nouveau, comme l'atteste le dossier du Monde diplomatique publié en 1997, mais la médiatisation de cet objet a en quelque sorte oblitéré l'approche scientifique du phénomène. La première synthèse relative aux questionnements reliant la mondialisation à l'Afrique n'est parue que très récemment (Les Temps modernes 2002).

De façon plus générale, les recherches actuelles indiquent que le terme de «mondialisation » apparaît comme polysémique et que les différentes définitions données ne semblent ni fixées, ni consensuelles (Stewart 1996). Pierre Bourdieu (2002) refusait l'emploi de ce terme, car parler « de mondialisation comme s'il s'agissait d'un phénomène naturel» ne permet pas de saisir cet objet sous l'angle de l'approche sociologique, et il proposait l'expression «politique de mondialisation». Que la «mondialisation » soit appréhendée comme la rencontre de normes idéologiques ou comme la manifestation de l'imposition de politiques néolibérales, l'expression une politique, même si elle a l'avantage de désigner un phénomène global, ne permet guère d'identifier et d'analyser de façon sectorielle le processus de mondialisation. On lui préférera donc celle de «politiques de mondialisation » qui indique la complexité et la multiplicité des relations induites et des champs investis. Par ailleurs, prendre en compte «les politiques de mondialisation » autorise l'identification des différents acteurs sociaux et leurs rôles respectifs. L'étude de ces politiques permet également de saisir où et comment s'élaborent les nouvelles normes, quels sont les acteurs producteurs de ces normes et comment celles-ci circulent et s'imposent dans le champ éducatif.

Le champ éducatif africain, à l'instar des systèmes éducatifs des pays du Nord comme de ceux de l'ensemble des pays du Sud, est atteint par les politiques de mondialisation (Brock-Utne 1996; Carnoy 1999; Lange 2001 ; Laval \& Weber 2002 ; Vinokur 2002). Les années 1990 peuvent 
apparaître comme emblématiques de la diffusion et de l'imposition de ces politiques. Dans la première partie de cet article, nous tentons de montrer le rôle joué par les conférences internationales dans la production et la diffusion de nouvelles normes scolaires. Les réformes scolaires financées par l'aide internationale sont décryptées à partir des moyens mis en place en vue de leur imposition et des conséquences observées. Dans la seconde partie, nous analysons la permanence des structures de pensée et des représentations sociales de l'École et de l'éducation. En dépit d'un phénomène brutal d'imposition de nouvelles normes et d'un consensus apparent et global, on constate que les relations famille/École demeurent à la fois ancrées dans des procès de reproduction sociale et culturelle, et projetées dans des évolutions autonomes, dont il est encore difficile d'en identifier le sens et le devenir. Finalement, le nouvel ordre scolaire mondial qui semble s'imposer n'est-il pas aussi très largement déformé par des stratégies sociales mêlant adhésion et contournement, rejet et récupération?

\section{Les années 1990 : véhicules et formes des politiques de mondialisation}

L'évolution des systèmes éducatifs africains est marquée par des rencontres successives d'abord provoquées par les conquêtes arabo-musulmanes, puis par les conquêtes coloniales européennes. Le développement de l'École africaine est encore très dépendant de ces rencontres qui l'ont fait naître : l'organisation des cycles d'enseignement, des contenus, des modalités de sélection et le choix de la langue d'enseignement découlent de cette histoire singulière. La dualité de certains systèmes scolaires d'une partie de l'Afrique, juxtaposant un système de type européen à un système de type arabo-musulman, montre de façon crue que les rencontres passées sont encore en cours de gestation, négociées et réinvesties selon les régions, les groupes sociaux et les pays ${ }^{1}$. Les nouvelles normes imposées dans le cadre des politiques de mondialisation viennent en quelque sorte se greffer sur des pratiques scolaires déjà diversifiées, complexes et en pleine mutation. Ce qui peut apparaître comme nouveau n'est en fait que la poursuite d'un processus continu de phénomènes liés de rencontres, d'imposition, de négociation, de rejet et d'appropriation. La spécificité des années 1990 tient essentiellement à deux faits nouveaux : d'une part, la volonté des différents acteurs d'influencer ce processus de rencontre et d'imposition de normes, et, d'autre part, l'agrégation à la fois d'une grande partie des pays « riches »

1. Tous ces pays (Burkina Faso, Cameroun, Mali, Niger, Sénégal, Tchad...) rencontrent des difficultés pour gérer cette dualité, entre tentatives de reconnaissance des écoles franco-arabes et des médersas ou tentatives d'incorporation dans le système éducatif national, tentatives de contrôle ou de marginalisation. Sur ce thème, en ce qui concerne le Mali, voir Louis BrenNer (2000). 
(que ce soit par l'entremise de leurs États ou de leurs administrations décentralisées, ou encore d'ONG internationales, émanation des représentations et aspirations des sociétés européennes vis-à-vis du continent africain), formant un conglomérat actif et plus performant que ne le pouvaient être (ou ne le voulaient être) les États-nations européens dispersés ou les dynamiques conquérantes - et parfois concurrentes - des communautés arabo-musulmanes. En effet, le consensus global construit par les États riches (et leurs ressortissants), constitué en agglomérat face au continent africain, est relativement récent; il découle directement de la fin de la disparition des deux blocs occidentaux, libéral et communiste, et du renoncement des anciennes métropoles coloniales à certaines de leurs prérogatives.

\section{Conférences internationales et essor d'un «partenariat » exclusif}

Si le développement de la scolarisation a débuté dès l'époque précoloniale, c'est la mise en place des administrations coloniales qui établit les bases institutionnelles d'un système scolaire. L'École coloniale se construit selon des schémas différents, en fonction de l'identité du colonisateur (allemand, anglais, belge, espagnol, français, portugais). Dès la période précoloniale, on observe des représentations familiales de l'École très diversifiées, certaines populations adhérant à l'École et suscitant l'ouverture de petites écoles primaires (Lange 2000). La demande d'éducation est alors déterminante dans l'instauration de ce nouveau mode de transmission des connaissances, de garde et de socialisation des enfants que constitue l'École de type européen. Cette diversité des représentations se prolonge au cours de la période coloniale où le développement de l'éducation demeure limité à la fois par des politiques d'éducation restrictives et par des stratégies familiales réservées face à l'École. En dépit de quelques progrès, il faut attendre les indépendances pour assister au développement très rapide des systèmes scolaires africains: les années 1960-1980 constituent une période d'euphorie et d' « explosion » des effectifs des différents degrés d'enseignement. Ce développement rapide est remis brutalement en cause au cours des années 19801985, marquées par la crise économique et financière ${ }^{2}$ qui secoue l'ensemble

2. La crise financière du début des années 1980 et la mise en place des Programmes d'ajustements structurels (PAS) a eu des conséquences néfastes sur le développement de la scolarisation : plusieurs centaines de milliers d'enfants ou de jeunes Africains ont ainsi été exclus de toute éducation scolaire ou ont interrompu précocement leurs études. Les politiques imposées dans le cadre de ces PAS ont une lourde responsabilité dans l'apparition des phénomènes de déscolarisation. Par ailleurs, elles sonnent le glas de l'influence des organisations onusiennes dans le champ éducatif au profit de la Banque Mondiale. Rappelons que c'est à partir des programmes de «la dimension sociale» des PAs que la Banque Mondiale commence à intervenir dans l'éducation des pays africains; cette intervention se poursuit et s'accroît par le biais des plans décennaux de l'éducation ou des programmes de lutte contre la pauvreté. 
des pays africains. Cette crise se caractérise du point de vue scolaire par un recul de la scolarisation, attesté dans un grand nombre de pays africains par la chute des effectifs scolaires et des taux de scolarisation. Cette « déscolarisation » de l'Afrique (Lange 1987, 1998) aura pour conséquence l'intervention accrue des bailleurs de fonds ${ }^{3}$ qui tenteront de contrecarrer cette tendance par une augmentation des aides financières destinées à l'éducation. On observe aussi le retrait des institutions onusiennes (Unesco) au profit des institutions financières (Banque Mondiale), ce qui n'est pas sans conséquence sur les orientations des politiques d'éducation des pays africains ${ }^{4}$. Caractérisées par l'afflux des financements extérieurs, les années 1990 marquent une nouvelle étape définie par un essor de la scolarisation en Afrique (à l'exception des pays touchés par les guerres civiles). D'une part, les effectifs scolaires enregistrent une hausse importante dans certains pays (Mali, Sénégal, Togo...), d'autre part, un nombre important de réformes est imposé par le biais de ces financements extérieurs.

La Conférence mondiale sur l'éducation pour tous (Jomtien, mars 1990) a ainsi suscité de nombreuses initiatives, tant de la part des pays du Sud que des bailleurs de fonds (institutions multilatérales, coopérations bilatérales, organisations non gouvernementales) et a influencé les politiques d'éducation mises en place ces dernières années. L'élaboration d'une conception plus large de l'éducation et l'instauration d'un consensus autour de la priorité, à la fois éthique et économique, à accorder à l'éducation ont ainsi vu le jour. Le consensus international obtenu lors de cette conférence a influencé de façon variable les politiques scolaires des pays africains, mais, globalement, l'interventionnisme des divers bailleurs de fonds s'est accru à la faveur des engagements pris. La nécessité du partenariat a été clairement proclamée et la Conférence de Jomtien a établi le fait que l'éducation pour tous était une responsabilité sociale qui engageait la participation de tous les acteurs nationaux (publics, privés et associatifs) et qui exigeait l'engagement international des organismes de coopération. Cependant, force est de constater que les grands exclus de ce partenariat furent d'une part les familles (parents et élèves) et, d'autre part, les enseignants, et que le rôle des États

3. Sous cette expression, sont inclus l'ensemble des intervenants extérieurs, quel que soit leur statut - organismes internationaux (Unesco, Unicef, Banque Mondiale...), coopération multinationale (Europe, Ligue arabe...), coopération nationale ou décentralisée, ONG ou associations...). Depuis la Conférence de Jomtien (mars 1990), on désigne souvent l'ensemble de ces acteurs par l'expression de «partenaires de l'École». En Afrique francophone, cette expression «bailleurs de fonds » tend à disparaître au profit de celle — nouvelle — de «Partenaires techniques et financiers » (PTF) qui a le mérite d'indiquer le poids décisionnel de ces bailleurs de fonds dans l'élaboration des politiques d'éducation.

4. Rappelons que les organisations onusiennes (Unesco, Unicef...) fonctionnent selon des principes de représentation plus équitables permettant l'expression des différences, ce qui n'est guère le cas des institutions financières (Banque Mondiale). 
africains, en tant que producteurs de politiques publiques, a été affaibli, sinon annihilé.

En premier lieu, rappelons que l'évolution de l'offre et de la demande d'éducation est déterminée par l'influence respective des différents acteurs de l'éducation. Jusqu'à la fin des années 1970, dans la plupart des pays africains, on observait un face à face État-société où l'État jouait un rôle déterminant: l'École était souvent pensée «comme la chose de l'État» (Lange \& Diarra 1999). La remise en cause de cette situation s'affirme au cours des années 1990, marquées par l'idéologie libérale triomphante prônant le retrait de l'État et la généralisation de l'économie marchande (y compris dans le domaine de la production et de la transmission des savoirs) (Lange 2001 ; Laval \& Weber 2002) et redistribuant ainsi les cartes entre les différents acteurs. On peut alors identifier trois principaux acteurs, à savoir les différentes composantes de la société civile, les États et les bailleurs de fonds qui jouent dorénavant un rôle dans le développement du secteur scolaire. Parmi les différentes composantes de la société civile, citons les familles, les communautés, les représentants élus des collectivités territoriales, les enseignants, les associations, les entrepreneurs ${ }^{5}$. Le rôle et le poids des différents acteurs varient énormément d'un pays à l'autre, en fonction du système politique en place (démocratique ou non, centralisé ou décentralisé, etc.), de l'histoire des communautés qui composent les États et du niveau de dépendance des pays vis-à-vis des organisations financières internationales. Ces différents acteurs n'ont en effet ni le même rôle, ni la même influence dans le développement de l'offre et de la demande scolaires. L'influence de ces acteurs s'exerce sur différents plans : apport financier, rôle dans l'élaboration des politiques scolaires, au sein des stratégies scolaires...

Le partenariat promu lors de la Conférence de Jomtien, appréhendé comme source d'efficacité et de démocratie, nécessite que l'on confronte ces discours consensuels à la réalité des faits observés sur le terrain ; 1'émergence ou le rôle nouvellement imparti à ces acteurs doit être examiné au regard du sens et de la réalité du partenariat dans la sphère éducative. La notion de « partenariat » a été récemment introduite dans le champ éducatif. En effet, ce terme fleurit tout d'abord au cours des années 1970 dans la sphère économique et sociale, avant de pénétrer le vocabulaire du domaine scolaire. La notion de partenariat semble alors surgir pour répondre à des situations de crise ou pour atteindre des objectifs nécessitant l'intervention

5. Par entrepreneurs, nous entendons ici aussi bien les entreprises du secteur moderne que les entreprises artisanales. Les relations entre les systèmes scolaires et le milieu des entreprises ont très peu été étudiées dans les pays les plus pauvres du fait que l'attention a surtout été portée sur les entreprises du secteur moderne, minoritaires dans ces pays. L'influence des entrepreneurs sur les politiques d'éducation et sur leurs politiques de formation à l'intérieur des entreprises ou sur les liens qui les unissent aux différentes écoles publiques ou privées se limite souvent aux formations techniques et professionnelles. 
de partenaires multiples ${ }^{6}$. Le partenariat peut ainsi être défini comme une association d'acteurs qui, par leur action commune, peuvent se fixer des objectifs qu'individuellement ils ne pourraient atteindre. Le terme de "partenariat» est toutefois ambigu, puisque travailler en partenariat peut aussi signifier travailler ensemble, mais pas forcément avec les mêmes objectifs, même si les partenaires coopèrent et tendent vers un même but.

Ces définitions correspondent bien au processus engendré par la Conférence de Jomtien, mais nous devons nous interroger sur les modalités du partenariat engagé, d'une part, et sur l'identité des partenaires mobilisés, d'autre part, pour atteindre l'objectif de l'éducation pour tous. Le recours au partenariat intervient après trois décennies d'objectifs relatifs à la scolarisation primaire universelle et à l'alphabétisation des adultes, sans cesse réitérés, du fait des échecs antérieurs. La volonté de développer le partenariat correspond bien à une situation de crise, ou du moins elle intervient alors que la démocratisation des systèmes éducatifs semble se gripper: elle répond à la nécessité de tenir les engagements visant à l'éducation pour tous. Cependant, si l'ensemble des bailleurs de fonds ont bien été reconnus comme partenaires, on peut se demander si les populations, à savoir les familles, les élèves et les enseignants l'ont été également. Dès le départ, en dépit du recours à la nécessité du partenariat et de l'affirmation selon laquelle l'éducation pour tous est une responsabilité sociale qui engage la participation de tous les acteurs, il semble bien que les familles n'aient pas bénéficié de la même reconnaissance que les différents bailleurs de fonds ou que certaines ONG internationales. De fait, la Conférence de Jomtien s'est située essentiellement sur le plan du développement de l'offre éducative, ignorant de la sorte tant la demande familiale d'éducation que les rapports à l'École des populations ou leurs représentations des institutions éducatives. Comme l'observe Jacques Hallak, « La question de la demande aurait mérité d'être mentionnée pendant la Conférence mondiale sur l'éducation pour tous, mais les principales préoccupations des participants étaient alors l'offre (accès et équité) et la réussite de l'apprentissage. La Déclaration et le Cadre d'action de Jomtien partent de l'hypothèse que si l'offre est satisfaisante (sur les plans de la quantité, de la localisation et de la qualité), enfants et adultes iront à l'école et l'objectif de l'éducation pour tous sera atteint » (Hallak 1994 : 14). En réalité, ni les besoins, ni les desiderata des familles ou des élèves en matière éducative n'ont été pris en compte ${ }^{7}$. De fait, les planificateurs de l'éducation s'intéressent très peu à la demande d'éducation et, dans la majorité des cas, celle-ci n'est jamais perçue comme la résultante de stratégies éducatives élaborées à partir des représentations de l'éducation

6. En France, par exemple, la notion de «partenariat»n'est introduite dans la sphère éducative que tardivement, au cours des années 1980, en vue de mener à bien les actions liant école et quartier, formation professionnelle et entreprises ou les projets de Zones d'éducation prioritaires (ZEP).

7. De même, ni les revendications, ni même les propositions des enseignants n'ont été prises en compte. 
et de l'École construites par les élèves et leurs familles. La demande d'éducation est ainsi uniquement appréhendée en termes de démographie scolaire, présupposant que les familles n'élaborent aucune stratégie face à l'École comme l'indique le document de statistique pour l'éducation pour tous publié par l'Unesco (World Education Forum 2000).

La volonté de prendre en compte les partenaires de l'École n'a guère concerné ni les enseignants, ni les élèves et leurs familles. Pourtant, ces trois acteurs « de base » devraient être considérés comme étant au cœur du partenariat entre l'État et les populations. On constate donc que, dans la plupart des pays, la multiplicité des acteurs et des intervenants financiers a sans doute permis d'ignorer le principal acteur de l'éducation, à savoir la famille. Finalement, les familles n'ont été sollicitées qu'en vue d'une implication financière plus importante : «[...] Partout, si les lois sur l'enseignement obligatoire garantissent la gratuité, on observe de fait la disparition de cette gratuité et l'augmentation continue des frais d'inscription à l'école publique, les populations étant de plus en plus sommées d'investir dans la scolarisation de leurs enfants » (Lange 2001 : 9). En fait, la mise à l'écart de certains «partenaires » est sans doute la conséquence de la volonté de ne pas remettre en cause les grandes lignes des réformes éducatives dessinées par les décideurs. Le partenariat promu lors de la Conférence de Jomtien semble donc en premier lieu avoir consacré le retrait de l'État au profit d'acteurs de l'éducation ne possédant guère une légitimité démocratique, ce qui est relatif à la plupart des bailleurs de fonds. Ceux-ci, quel que soit leur statut — institutions financières (Banque Mondiale), coopérations bilatérales ou multilatérales, ONG internationales) ne disposent d'aucun mandat des populations pour lesquelles ils définissent les politiques d'éducation. Certains d'entre eux n'hésitent cependant pas à imposer ces politiques selon différents moyens, notamment par le biais de conditionnalités liées aux prêts ou aux dons qu'ils proposent aux pays les plus pauvres.

En conclusion, si les conférences internationales sur l'éducation ont toujours promu de nouvelles utopies ou idéologies, la spécificité des conférences des années 1990 tient à l'arrivée des institutions financières, à leur capacité d'influer sur le contenu et les décisions prises en finançant ces rencontres et les projets éducatifs acceptés lors de ces conférences, et de créer un consensus apparent. Le consensus global accepté repose sur la formulation de questions éthiques les plus élémentaires conditionnant l'adhésion de tous «qui est pour l'éducation pour tous? », excluant de fait les questions de fond «quelle éducation pour qui, avec qui et pour quel projet de société ? ». L'affirmation du partenariat, imposé comme gage d'efficacité et de démocratie, ne résiste pas à l'analyse de l'exclusion des enseignants et des familles, pourtant premier acteur de l'éducation et de la socialisation des enfants. Ce processus de planification de l'extérieur, présenté comme l'émanation et la volonté de tous, nie et oblitère les différences de pensée, ce qui explique qu'il est perçu comme processus de mondialisation. En affirmant le consensus et en excluant la parole de ceux qui pourraient s'y 
opposer, il se caractérise aussi par l'absence de débats, qui renvoie de fait à la négation des expressions démocratiques : l'imposition idéologique est source de diktats et les populations africaines ne sont perçues que comme réceptacle de politiques ou de réformes, produites pour leur bien-être, défini hors de leurs propres représentations.

\section{Aide internationale et réformes scolaires}

L'interventionnisme accru des pays du Nord dans la définition et la mise en œuvre et dans le financement des programmes éducatifs africains induit que les politiques d'éducation sont de plus en plus impulsées de l'extérieur selon un modèle unique, d'où une certaine uniformisation de ces réformes (voir le tableau page 152); les systèmes éducatifs tendent à se réformer et à se développer selon un même schéma. La dépendance financière varie selon les États africains, et les politiques éducatives de ces pays sont financées pour une part allant de $30 \%$ à $80 \%$ des sommes publiques destinées à l'éducation. Avec un budget éducatif financé à près de $80 \%$ par l'aide extérieure, le Tchad détient le record de la dépendance (Nomaye 2001). Ainsi, par-delà les différences historiques, économiques et politiques des pays, on semble de plus en plus assister à l'imposition d'un ordre éducatif mondial (Lange 1998 : 298 ; Lange 2001 : 6 ; Laval \& Weber 2002). Cependant, en dépit de cette uniformisation, force est de constater que les initiatives sociales en matière éducative promues par les sociétés civiles, tout comme les stratégies familiales d'éducation, restent déterminantes dans l'évolution des systèmes. Les années 1990 reflètent bien ce rapport ambigu à l'École : la dépendance accrue des pays africains face aux pays occidentaux (pratiquement aucun pays africain ne peut dorénavant financer son système scolaire sans les financements étrangers), stigmatisant l'imposition d'un ordre scolaire mondial, se conjugue avec des dynamiques sociales qui s'affranchissent des modèles étatiques. La diversification du champ scolaire ${ }^{8}$ africain est symptomatique des effets conjugués de l'imposition du dogme libéral prônant le retrait de l'État de la sphère éducative et des nouvelles modalités d'appropriation de l'École par les sociétés.

Dans les pays les plus pauvres, l'imposition des principales réformes (classes multigrades, double vacation, diminution des taux de redoublement, allègement des programmes, priorité affichée en faveur de la scolarisation des filles, perte du statut de fonctionnaire et salaires en baisse pour les enseignants, diminution du niveau académique des nouveaux enseignants

8. Ce que nous nommons « diversification du champ scolaire » correspond au processus du retrait de l'État, constaté par l'apparition ou le développement rapide à côté des écoles publiques (souvent très majoritaires, voire exclusives au début des années 1990, selon les pays) de nouveaux types d'école (écoles privées laïques ou confessionnelles, écoles communautaires ou associatives...) et la reconnaissance juridique de ces nouvelles écoles. 
recrutés, etc.), via les financements extérieurs, dont ceux de la Banque Mondiale en particulier, n'ont guère reçu l'aval des familles africaines, souvent obligées, lorsqu'elles en ont les moyens relationnels ou financiers, de contourner ces réformes. Quant aux enseignants, ils ne sont que rarement considérés comme des partenaires à part entière et sont le plus souvent perçus comme «un coût excessif et devant nécessairement être réduit », comme des «facteurs contraignants » ou comme des « obstacles » à la mise en place des réformes éducatives. L'imposition de ces réformes a un coût certain tant humain et financier qu'éthique : démobilisation du corps enseignant, stratégies d'évitement ou de refus des familles, rémunération d'experts internationaux coûteux, cadres des ministères de l'Éducation qu'il faut « motiver » à grands coups de per diem... L'élaboration des réformes éducatives devient un lieu de marchandage occultant le rôle sociétal et politique qui a autrefois présidé à leurs agencements ${ }^{9}$.

Ces réformes, après avoir été imposées dans le cadre du financement des «Plans pour l'éducation», sont dorénavant mises en place au sein des «Programmes décennaux ${ }^{10}$ ou de l'initiative «Pays pauvres très endettés » (PPTE) (Henaff dans ce numéro). Rappelons que ces plans et ces différents programmes réalisés ou en cours de réalisation sont élaborés par des commissions dans lesquelles siègent à la fois des cadres nationaux et un consortium de bailleurs de fonds. Si les études ou débats préalables à l'élaboration de ces programmes peuvent laisser penser qu'ils émanent d'une volonté à la fois gouvernementale et sociétale ${ }^{11}$ et qu'ils sont simplement financés par l'extérieur, l'uniformisation des différents plans africains et le degré accru de dépendance financière des États africains semblent bien confirmer l'extériorité des réformes mises en place. Outre que ces pays sont dépossédés de leur autonomie en matière de politique scolaire et soumis au diktat des bailleurs de fonds, la perte de décision en matière éducative produit divers effets. Le premier s'observe dans le fonctionnement de la mise en place et de la gestion des politiques scolaires. Les bailleurs de fonds imposent une programmation par étapes qui rend difficile l'élaboration de

9. Voir par exemple, le rôle qu'ont tenu les États généraux de l'éducation, lors des Conférences nationales tenues en Afrique, durant la période de transition démocratique, dans l'appropriation de l'École par les populations. Or, sans cette appropriation, les échecs des politiques d'éducation s'avèrent patents.

10. Les «Plans décennaux pour l'éducation» ont été définis en Afrique selon les mêmes méthodes, les mêmes principes et les mêmes difficultés, en dépit des variantes nationales pour les dénommer (par exemple, Programme décennal de développement de l'éducation (PRODEC) au Mali ou Plan décennal de développement de l'éducation de base (PDDEB) au Burkina Faso).

11. Par exemple, dans le cas des Plans décennaux, des enquêtes d'opinion ont été menées, officiellement pour prendre en compte les desiderata des populations. Cependant, dans les faits, les revendications essentielles des familles ont été systématiquement ignorées lorsqu'elles s'opposaient aux politiques préconisées par les bailleurs de fonds les plus influents. 
EXEMPLES DE QUELQUES RÉFORMES (ET DE LEURS INITIATEURS) EN AFRIQUE

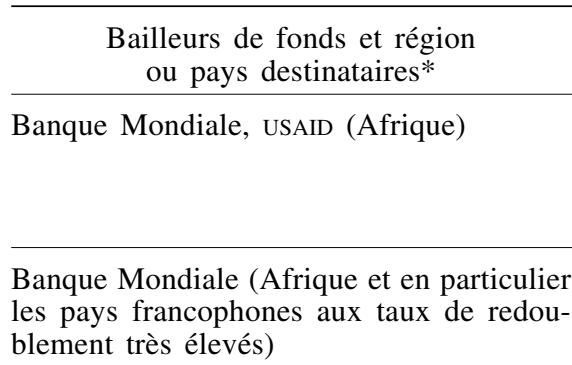

Banque Mondiale, USAID (Afrique)

\section{Réformes}

Double vacation (imposition: $40 \%$ des classes devront être en DV) ; financement d'une partie des primes attribuées aux enseignants en poste dans des classes à DV

Limitation des redoublements (maximum $15 \%$ de redoublants de la $1^{\text {re }}$ à la $5^{\mathrm{e}}$ année d'école)

Priorités financières à l'enseignement primaire (rééquilibrage du budget destiné au secteur éducatif en faveur de l'enseignement de base)

Scolarisation des filles (campagne de sensibilisation, quotas imposés dans les écoles non formelles, aides ponctuelles en faveur de la scolarisation des filles...)

État malien (lancement des classes expérimentales en langues nationales)

Belgique (lancement de la méthodologie convergente) (Mali)

USAID, UNICEF (financement de la pédagogie convergente) (Mali)

Coopération suisse (financement des écoles bilingues) (Burkina Faso)

CILS (Pays du Sahel)
Banque Mondiale, USAID (Afrique)

Enseignement environnemental

Limitation des bourses destinées aux lycéens et étudiants, «conditionnalité » souvent imposée aux pays pour obtenir des fonds destinés à l'éducation primaire

Pays arabes (pays d'Afrique où résident des musulmans)

Individus, Banque Mondiale, Fonds pour l'éducation (Afrique)

Fonds pour l'éducation, France, Allemagne, associations et ONG internationales, associations nationales, communautés villageoises, associations de quartier, associations de migrants (Afrique)

ONG internationales, programmes des Écoles non formelles (aide à la création et Nations Unies (Afrique)

Financements via les projets de décentralisation ou les plans décennaux de l'éducation (Afrique) au fonctionnement)

Décentralisation du système scolaire et redistribution des actions éducatives entre l'État et les collectivités territoriales

\footnotetext{
* Sont indiqués entre parenthèse les pays d'Afrique concernés.
} 
politiques sur le long terme et le suivi des opérations, et conduit à des prises de décision par à-coups, souvent déconnectées des situations réelles. Le second effet est induit par le fait que la réussite doit être au rendez-vous de chacune de ces étapes, car de cette réussite dépendent les futurs financements. Les effets pervers de ce type de fonctionnement apparaissent alors crûment : contraints de réussir pour justifier la bonne utilisation des financements accordés, les pays sont tentés d'investir dans les zones déjà favorisées, où les projets antérieurs ont connu des résultats positifs ${ }^{12}$, ce qui a pour conséquence immédiate l'accroissement des disparités et l'abandon de zones scolairement jugées difficiles. Autre effet pervers de ces financements sous conditions, les résultats des politiques mises en œuvre ne peuvent être que globalement positifs, et des instructions sont données dans ce sens aux différents agents du système scolaire. Il n'est donc plus possible d'effectuer l'évaluation de certains projets, et des documents officiels sont systématiquement «falsifiés » pour répondre aux attentes — supposées ou réelles des bailleurs de fonds ${ }^{13}$. La spécificité de ces réformes ou programmes financés par l'extérieur est d'échapper à toute évaluation (Dahou 2002), du fait de leurs enchaînements successifs, un programme financé par l'extérieur étant à son achèvement aussitôt remplacé par un autre.

Le découpage des réformes et leur prise en charge financière par les différents bailleurs de fonds sont parfois perçus par les parents d'élèves comme des décisions arbitraires, seulement motivées par les objectifs gouvernementaux de capter la manne financière qui accompagne ces réformes. Les enseignants les rejoignent souvent dans cette analyse et sont réticents à appliquer des réformes dont la poursuite, et donc la pérennité, dépendent, de façon aléatoire, du renouvellement des financements extérieurs. Parfois, leurs revendications portent explicitement sur le rôle que l'État doit jouer en légiférant pour donner un cadre officiel aux réformes en cours ${ }^{14}$. Dans

12. C'est le cas par exemple du Mali où la presque totalité des financements du $\mathrm{IV}^{\mathrm{e}}$ plan d'éducation, financé par un groupe de bailleurs de fonds (Banque Mondiale, Coopération française, USAID, etc.), ont été dirigés vers les régions les plus favorisées du point de vue économique et scolaire, où la demande scolaire préexistait ainsi que les infrastructures, et où les autorités étaient sûres de pouvoir facilement augmenter le taux de scolarisation.

13. Tel responsable d'un service statistique s'est ainsi vu convoqué pour calculer le taux de scolarisation selon « une nouvelle formule », qui, de fait, permettait d'augmenter de façon substantielle le taux de scolarisation, augmentation destinée à montrer aux bailleurs de fonds que l'argent qu'ils avaient investi dans l'éducation avait produit les effets escomptés. Il devient alors très difficile au chercheur de naviguer entre les chiffres qu'on nous assure «pas encore redressés politiquement », ceux qui sont effectivement « redressés », et les données volontairement «floues » qui n'autorisent plus aucune interprétation. Certains services statistiques renoncent dorénavant à calculer les taux nets de scolarisation, préférant afficher des taux bruts (plus élevés du fait du mode de calcul).

14. C'était, par exemple, l'une des revendications des enseignants maliens à propos de la «pédagogie convergente » (voir le tableau page 152), dont l'expérimentation a été financée durant six ans par des fonds belges, puis américains, et dont la survie fut assurée par des fonds de l'Unicef. Qu'ils soient opposés ou favorables à 
le face-à-face États-sociétés viennent se glisser des acteurs (représentants des institutions internationales, des coopérations nationales ou des ONG) qui travestissent les relations qu'États et populations essaient de construire. Cette situation est particulièrement inquiétante dans les pays ayant connu un processus de démocratisation, où le poids financier des intervenants étrangers transforme souvent des rapports citoyens en rapports marchands.

L'implication des cadres nationaux dans les projets financés par l'extérieur posant souvent problème, les intervenants étrangers sont dorénavant obligés de contourner cette réticence par l'attribution d'avantages financiers, symboliques ou matériels. Toute réforme scolaire comporte ainsi des stages où les per diem constituent un complément de salaire (nécessaire au consensus), parfois suivi de l'attribution de primes permettant de «motiver» les fonctionnaires (prime attribuée pour «la double vacation », pour « les classes multigrades », pour «l'enseignement environnemental », etc.). L'inertie des ministères de l'Éducation est contournée par la multiplication des structures parallèles de gestion des différentes réformes ${ }^{15}$, qui nuit à la cohérence de la gestion administrative et participe au discrédit des structures étatiques. Enfin, les différents «projets» captent une partie des meilleurs cadres de l'administration, soit en les débauchant de la fonction publique (ONG), soit en les accueillant le temps d'un financement au sein de leurs structures (organisations internationales) $^{16}$.

\section{Vers un nouvel ordre scolaire?}

Ce désengagement progressif de l'État a été accentué depuis quelques années par l'application des mesures visant à accroître la participation de tous les acteurs et partenaires de l'École. On observe depuis lors la mise en place d'une politique d'éducation qui fait appel à ces acteurs et partenaires de l'École pour qu'ils s'impliquent davantage dans la gestion et la prise en charge des problèmes d'éducation de leurs localités. Or, cette politique a entraîné une explosion d'initiatives privées et/ou communautaires en matière de création d'écoles formelles ou non formelles (Esquieu \&

cette réforme, les enseignants dénoncent l'attitude de l'État, incapable de jouer son rôle de législateur et de gestionnaire (absence de décret, d'arrêté ou simplement de circulaire...). En clair, les réformes promues par les financements extérieurs se trouvent en dehors de tout cadre législatif national : elles relèvent en quelque sorte d'une extraterritorialité remettant en cause la souveraineté des Etats.

15. Le cas le plus récent est celui qui porte sur les projets liés à la scolarisation des filles, où l'inflation du nombre de cadres responsables de la scolarisation des filles grève les budgets destinés à l'aide, sans que l'efficacité de ce mode de fonctionnement soit pour l'instant convaincante.

16. Entre 1994 et 1998 (période de nos enquêtes au Mali), plusieurs services du ministère de l'Éducation de base du Mali se sont ainsi vus décapités par l'intégration de leurs responsables au sein des représentations locales des organisations internationales. 
Péano 1994 ; Lange \& Diarra 1999 ; Chediel et al. 2000 ; Cissé et al. 2000 ; Diarra et al. 2000 ; Gbogbotchi et al. 2000). Face à l'explosion des initiatives privées (associations, ONG nationales ou internationales, coopérations bilatérales, communautés, etc.), certains pays commencent à percevoir les dangers que constitue ce mouvement centrifuge incontrôlé et tentent de recenser, d'organiser et d'uniformiser les différentes initiatives dans un mouvement centripète qui, pour l'instant et en l'absence de moyens financiers adéquats, ne semble pas pouvoir opposer une force suffisante.

Il est évident que les choix des intervenants étrangers conjugués avec l'avènement de la démocratie observé dans certains pays africains (Mali, Sénégal...) a permis de libérer les initiatives privées ou communautaires, et que la dynamique sociale remarquée précède le plus souvent les décisions ou décrets gouvernementaux ${ }^{17}$. Aujourd'hui, non seulement la demande sociale d'éducation peut s'exprimer, mais elle est aussi en mesure d'impulser et même de gérer l'offre scolaire par la création de nouvelles écoles (communautaires, associatives...). Cependant, la mesure de l'impact réel de ces écoles, en termes d'effectifs scolarisés, de réussite scolaire et de satisfaction de la demande des parents, demeure encore embryonnaire du fait que le phénomène est encore mal connu ${ }^{18}$. De même, les problèmes rencontrés, tant au plan de la gestion de ces nouvelles écoles que de celui du devenir social des enfants qui les fréquentent, n'ont pas été identifiés. La principale question reste celle de l'évaluation des limites ou des entraves au développement de ces écoles et de la pérennité de l'ensemble des différentes écoles créées. Répondre à cette question, et à celles qui suivent, consiste aussi d'une certaine façon à mesurer la validité du discours dominant sur les bienfaits supposés du retrait de l'État dans le secteur éducatif. Peut-on augmenter le taux de scolarisation de façon substantielle grâce à la création de ces écoles? Quel sera le devenir de ces écoles, en grande partie financées par les parents les plus démunis ? Par ailleurs, en se dégageant du secteur scolaire, d'une certaine façon, les États renoncent au dialogue avec les populations, dont les rapports à l'École et les représentations sociales de l'éducation demeurent à la fois inconnus et distants des modèles universels imposés par le nouvel ordre mondial.

17. Ainsi, au Mali, la majorité des écoles communautaires ont été créées avant l'adoption de la loi de 1994 portant statut sur l'enseignement privé et du décret de la même année fixant les modalités d'organisation et de fonctionnement des écoles communautaires. Ces «nouvelles écoles » changent d'ailleurs de nom lorsqu'elles sont reconnues par l'État: les écoles spontanées du Tchad deviennent des écoles communautaires, les écoles pirates du Burkina Faso des écoles privées, les écoles clandestines du Togo des écoles d'initiatives locales, etc.

18. Par exemple, on ne connaît pas le nombre exact des écoles franco-arabes ni des médersas « privées », ni même des écoles créées par les communautés non déclarées officiellement ou non reconnues par les États, les statistiques scolaires ne prenant en compte que les écoles ayant reçu un agrément de l'État. 


\section{Des relations famille-École ignorées}

Dix ans après la Conférence de Jomtien, les échecs ont été analysés lors du Forum de Dakar: "Quels ont été les échecs? Aucun des objectifs n'a été pleinement atteint, même si de grands progrès ont été accomplis pour certains. Quelques régions ont connu des difficultés, voire un recul, par exemple en matière de scolarisation et de participation dans le primaire, d'égalité des sexes ou de réponse aux besoins des groupes socialement défavorisés. » (Forum mondial sur l'éducation 2000 : 65). Il n'est pas sûr cependant que le poids des stratégies familiales ou que les relations famille-École ont été bien pris en compte. Pourtant, constater que le développement de la scolarisation n'est ni linéaire ni réparti équitablement selon les régions ou les groupes sociaux indique bien que l'offre d'éducation ne détermine pas seule l'évolution de la scolarisation. De même, et de façon encore plus évidente, les inégalités scolaires entre filles et garçons ne peuvent uniquement s'expliquer par l'offre d'éducation : ici, la demande d'éducation joue souvent le premier rôle.

Pour comprendre les expressions et les significations de la demande familiale d'éducation, il est nécessaire d'étudier les représentations de l'École. En effet, l'étude des représentations sociales ou familiales autorise le passage de l'analyse descriptive (par exemple, constat de la baisse des effectifs scolaires dans une région où l'offre se maintient, ou encore faible scolarisation des filles, alors que l'offre ne semble pas ségrégative) à l'analyse explicite qui permet d'effectuer le lien entre les faits, les intentions et les pratiques. On peut définir les représentations sociales comme un acte de pensée par lequel un acteur se rapporte à un objet (Jodelet 1991). Lorsqu'un acteur se représente un objet - ici en l'occurrence l'École - il le reconstruit et l'interprète pour le rendre intelligible selon ses propres connaissances et ses appartenances sociales et culturelles : les représentations sociales se forment, s'ordonnent et s'opposent au sein de processus d'interactions multiples.

Les représentations familiales de l'École dépendent donc étroitement des relations qui s'instaurent entre les familles et l'École (Henriot-Van Zanten 1988). Ces relations famille-École relèvent de deux sphères principales qui dépendent l'une de la participation individuelle, l'autre de la participation collective. La première renvoie au degré d'articulation entre le processus de socialisation initié par la famille et celui mis en œuvre par l'École, d'une part, et aux différentes modalités de l'accompagnement familial de la scolarité, d'autre part. La seconde est celle de la participation collective des familles au fonctionnement des établissements scolaires, notamment à travers leurs représentants associatifs (associations de parents d'élèves, comités de gestion de l'école...).

Le degré d'articulation entre le processus de socialisation initié par la famille et celui mis en œuvre dans la scolarisation d'un enfant dépend en grande partie de la proximité culturelle que la famille entretient avec 
l'École. Cette proximité est liée à l'histoire scolaire et sociale de chaque famille : fréquentation scolaire ou non de ses membres, réussites et échecs passés, résultats économiques (telles que l'insertion ou non dans le monde du travail et la réussite économique et financière) ou résultats sociaux et familiaux (telles que la cohésion ou la désunion de la famille) induits par le procès éducatif engagé. L'accompagnement de la scolarité des enfants et des jeunes découle également des représentations. Cet accompagnement qui dépend étroitement du degré d'adhésion au projet éducatif et sociétal de l'École est déterminant pour la réussite scolaire. Il inclut en premier lieu les relations enseignants-familles ${ }^{19}$ qui déterminent en grande partie le style d'accompagnement de la scolarité des enfants par les familles, tels que le refus ou l'acceptation des demandes des enseignants (en assiduité, en respect des horaires et des règles internes à l'école, en fournitures scolaires...). Le style d'accompagnement de la scolarité se caractérise aussi par l'espacetemps accordé à l'enfant au domicile pour lui permettre d'effectuer les tâches scolaires demandées, par la valorisation ou non des savoirs acquis par l'enfant à l'école, par l'intérêt porté sur ses résultats scolaires, etc. Bien sûr, toutes les familles ne peuvent mobiliser les mêmes moyens et la même énergie pour accompagner la scolarité de leurs enfants, mais à ressources égales, on peut constater de très grandes différences qui permettent de mesurer le degré d'adhésion au projet éducatif et sociétal de l'École.

La participation collective des familles au fonctionnement des établissements scolaires s'exprime à travers les associations de parents d'élèves ou des comités de gestion de l'école. Elle dépend souvent du niveau de centralisation ou de décentralisation du système scolaire, les systèmes très centralisés ayant tendance à écarter les familles de la gestion des écoles. Lorsque cette participation est effective, on observe cependant que tous les parents ne sont pas représentés au sein des associations ou des comités de gestion. Les parents les plus instruits ou ceux qui appartiennent aux classes sociales les plus favorisées s'investissent généralement beaucoup, tandis que les familles les plus démunies sont absentes ou jouent un rôle secondaire. De même, dans la plupart des pays africains, les femmes ne sont guère impliquées dans ces structures associatives. Le désintérêt envers ces associations peut relever soit de l'ensemble des parents d'élèves, soit de certains groupes. En l'absence de pouvoir réel de ces associations ou/et de fonctionnement

19. Les relations enseignants-familles apparaissent souvent déterminantes dans l'élaboration des représentations familiales de l'École. La marginalisation politique des enseignants par les gouvernements autoritaires, la remise en cause de leur statut socio-économique par les politiques néolibérales contribuent au rejet de l'institution scolaire qui n'apparaît même plus aux yeux des populations comme assurant un minimum de respect et de conditions de vie décentes aux enseignants, et donc sera, à ne pas en douter, incapable d'assurer quoi que ce soit à leurs propres enfants. Ainsi, prôner la baisse des salaires des enseignants, dans le but de pouvoir en recruter en plus grand nombre afin de développer l'offre, peut s'avérer complètement contre-productif, si les représentations familiales en sont affectées de telle sorte que la demande d'éducation faiblit (LANGE 1998). 
démocratique, les parents renoncent très vite à toute participation collective, ce qui peut nuire à la dynamique scolaire, et l'absence de porte-parole émanant des familles constitue un obstacle au dialogue entre l'État ou les collectivités territoriales et les parents d'élèves.

Les perceptions de l'École par les familles dépendent aussi du niveau de scolarisation atteint par les différents pays. Plus un pays est scolarisé et plus l'École est instituée, plus l'adhésion des familles au procès de scolarisation est grande et plus les représentations de l'École «s'unifient ». Ce qui n'empêche pas de constater à l'intérieur d'un même pays une très grande variété des représentations et des rapports à l'École. Ces rapports à l'École doivent obligatoirement être contextualisés : analyser ces rapports et les représentations qu'ils engendrent en dehors des contextes économiques, sociaux et politiques s'avère impossible du fait que les rapports à l'École sont sous-tendus par les conditions de vie des populations. La demande sociale d'éducation, et donc les stratégies familiales face à l'École, repose sur les représentations que les familles se font de l'éducation et de l'École. Du refus de l'École à l'acharnement scolaire, les modulations des rapports à l'École sont très importantes. Rien n'est jamais acquis, et le rapport à l'École est en quelque sorte sans cesse négocié, en fonction du propre vécu des familles et de l'évolution des conditions socio-économiques et politiques. Dans les pays les plus pauvres, et au sein des groupes les plus défavorisés, l'instabilité des rapports à l'École constatée reflète l'insécurité sociale, économique et politique que subissent ces populations ${ }^{20}$.

La famille, lieu premier d'éducation des enfants, est aussi l'institution qui pense le plus l'École, qui a le plus à dire sur l'École, mais, paradoxalement, c'est aussi l'institution la moins consultée, celle que les politiques scolaires ignorent le plus souvent. Entre obligation et exclusion scolaires et politiques scolaires imposées, la place laissée au dialogue et la prise en compte des desiderata des familles demeurent à la fois marginales et souvent limitées à de courtes périodes, historiquement circonscrites à des phénomènes de démocratisation de la vie politique. De fait, les connaissances que nous avons des représentations familiales de l'École sont très parcellaires, factuelles et rarement prises en compte tant par la littérature des organismes internationaux que lors de l'élaboration des politiques nationales d'éducation.

Cette méconnaissance des représentations familiales ou sociales de l'École — et de l'éducation —, du rapport aux savoirs renvoie à l'idéologie scolaire dominante qui pense le développement éducatif des populations comme relevant uniquement de l'offre. L'École peut aussi être parfois perçue comme processus exclusif d'éducation et de socialisation, négation

20. Cependant, même dans les pays « riches » du Nord, on peut observer des revirements spectaculaires de la demande sociale d'éducation, comme par exemple en France, où après une période de croissance rapide des effectifs de l'enseignement secondaire et supérieur, nous observons actuellement une stagnation, voire un début de «déscolarisation » dans l'enseignement supérieur. 
d'une réalité où la socialisation des enfants des pays africains s'opère le plus souvent en dehors de l'École (Lange et al. 2002). Alors qu'un consensus autour de la nécessité du partenariat pour parvenir à l'éducation pour tous s'est établi lors de la Conférence de Jomtien, les soubassements de l'exclusion des familles de ce «partenariat » et les raisons qui sous-tendent la non-reconnaissance des parents et des élèves comme acteurs de l'éducation relèvent sans doute d'une méconnaissance et du refus de reconnaître les différences des représentations de l'École. On note cependant que, lorsqu'il y a convergence entre les stratégies familiales et les bailleurs de fonds, la reprise d'initiative en matière éducative et scolaire des familles est accompagnée, comme dans le cas de l'émergence de nouveaux types d'écoles (écoles des parents, écoles communautaires...).

\section{Des représentations sociales de l'éducation autonomes}

Le droit à l'éducation pour tous (Unesco 2000) affirmé lors de la Conférence de Jomtien a obtenu un large consensus de la part des États africains, mais, paradoxalement, les populations ne semblent guère avoir été consultées. Personne ne semble d'ailleurs s'être seulement demandé comment les populations perçoivent ce droit ${ }^{21}$. Les rares enquêtes de terrain indiquent que la conception du droit à l'éducation pour tous n'est guère universelle. L'étude des représentations familiales du droit à l'éducation pour tous révèle que ce droit n'est jamais perçu comme un droit déconnecté des autres droits. Ainsi il est souvent cité comme secondaire par rapport au droit à la santé par les parents d'élèves et les enseignants, ou comme nécessaire et condition préalable à la réalisation du droit à l'éducation ${ }^{22}$. Cependant, ce sont surtout les femmes, bien plus que les hommes, qui semblent concevoir l'éducation comme un "droit » au sein d'un ensemble de « droits » complémentaires entre eux. Ainsi leur centre éducatif idéal serait relié à des activités rémunératrices, disposerait d'un point d'eau, serait adjacent à une infirmerie et à une maternité, et l'école primaire des enfants serait proche..., ce qui renvoie aux droits à l'autonomie financière, à la santé, à l'éducation, à l'École... C'est en quelque sorte leur conception du développement social et économique qui met bien en relief la difficulté des politiques sectorielles en vigueur pour répondre aux besoins fondamentaux des femmes. Du fait de leurs problèmes de santé spécifiques (maternités, soins des enfants), de

21. À l'exception de quelques travaux en cours comme ceux dirigés par l'Association pour la promotion de l'éducation non formelle au Burkina (APENF-Burkina Faso), l'Institut interdisciplinaire d'éthique et des droits de l'Homme et la Chaire d'histoire et de politique économiques de l'Université de Fribourg (Suisse).

22. Alors que les rapports sur l'éducation émanant des organismes internationaux mettent l'accent sur la nécessité de l'éducation comme gage d'amélioration du niveau sanitaire des populations (et donc comme investissement rentable), les familles et les enseignants rappellent qu'il faut d'abord être en bonne santé pour être scolarisé ou pour fréquenter les centres d'alphabétisation. 
leur besoin d'autonomie économique (recherche d'activités rémunératrices), et de la surcharge de travail liée à leur sexe (tâches ménagères qui s'additionnent aux tâches éducatives et aux tâches productives), les femmes ne peuvent percevoir une amélioration de leurs conditions de vie que si leurs besoins élémentaires sont couverts conjointement (accès à l'éducation, aux ressources financières, aux soins, à l'eau...). Vouloir promouvoir le droit à l'École ou à l'éducation sans prendre en compte tous les manquements aux autres droits risque de ne pas engendrer l'adhésion populaire présumée et/ ou souhaitée.

Si les représentations du droit à l'éducation des familles urbaines sont très diversifiées parce que fonction des catégories socioprofessionnelles et de l'environnement culturel, celles des familles rurales semblent peu diversifiées. À l'exception des zones rurales scolarisées de longue date et dont les populations ont incorporé la culture scolaire, le droit à l'éducation est souvent perçu de façon presque exclusive comme un droit positif, mais en quelque sorte collectif, donc non individualisé. Ce collectif ne renvoie pas à l'ensemble des enfants ou des jeunes, mais plutôt comme devant être collectivement utile à l'ensemble de la communauté, même si une minorité d'enfants est scolarisée. Ainsi, l'intérêt de l'enfant n'est jamais pris en compte et n'est que rarement mentionné. Le droit à l'éducation ou le droit à l'École est perçu comme le droit de la famille ou de la communauté villageoise, et les bénéfices tirés de l'exercice de ce droit semblent ne pouvoir être appropriés que par la collectivité. Cependant, au sein de la collectivité, ce sont essentiellement les adultes (plus précisément les chefs de famille) et en particulier les hommes qui semblent être les bénéficiaires attendus des effets de la fréquentation de l'école ou des centres d'alphabétisation. L'éducation des filles est ainsi perçue positivement si les filles respectent leurs aînés et « qu'on peut les marier sans difficulté » (sous-entendu, que les filles acceptent le mariage proposé), ce qui explique que les modules sexuellement spécifiques (cours de cuisine, de couture...) introduits dans certaines écoles expérimentales ou dans les centres d'alphabétisation rassurent les familles des élèves. Le droit à l'éducation n'est ainsi jamais relié ou mis en relation avec les droits des femmes et les droits des enfants. Ceux-ci ne sont d'ailleurs pas perçus comme « individus », capables de faire usage de droits. Or, quelle que soit la valeur morale intrinsèque à cette conception du droit à l'éducation, cette représentation nuit à la scolarisation ou à la formation du plus grand nombre, puisque l'éducation pour tous est en quelque sorte conçue comme l'éducation de quelques-uns au profit de tous (Compaoré \& Lange 2002).

Ces représentations de l'éducation pour tous rendent intelligibles les stratégies éducatives des familles rurales africaines qui consistent à ne choisir que quelques enfants ou quelques jeunes en vue de leur formation. Elles sont donc très éloignées des théories sociologiques considérant les acteurs sociaux comme des «consommateurs d'école». Ces théories de l'École, subordonnées à une apologie de «l'homo oeconomicus » et de la liberté, 
qui réduisent la notion de stratégie à des pratiques de consommation individuelles, socialement décontextualisées, sont cependant implicitement défendues par la plupart des «Partenaires techniques et financiers » qui influencent, à partir de ces présupposés, les politiques d'éducation africaines. L'étude des représentations familiales montre en quoi ces théories qui occultent les inégalités socioculturelles et qui n'envisagent que les stratégies individuelles, niant l'existence de stratégies collectives, ne peuvent être transposées en Afrique. Le rôle de socialisation de l'École infère que l'institution scolaire ne peut être un bien de consommation comme les autres; les stratégies éducatives ne répondent pas seulement à des besoins économiques et n'induisent pas non plus que des comportements sociaux : elles jouent un rôle dans la reproduction sociale et culturelle, elles s'inscrivent au sein de projets sociétaux.

Cependant, vouloir scolariser ses enfants ne suffit pas, encore faut-il le pouvoir. Les familles ne sont pas égales face à l'offre scolaire : le nombre de places disponibles, l'éventail du choix selon les différents types d'écoles (privé/public, religieux/laïc) et selon les degrés d'enseignement varient considérablement d'une région à l'autre, du milieu rural au milieu urbain. Elles ne sont pas égales non plus quant au capital économique ou relationnel qu'elles peuvent mobiliser en vue de la scolarisation de leurs enfants. Face à une offre donnée, les stratégies les plus diverses s'observent, et l'appropriation de l'École par les familles renvoie aux différents ancrages au sein des sociétés que nécessite cette institution. Du refus de la scolarisation, en passant par l'acceptation modérée, jusqu'à l'adhésion complète, le rapport à l'École se négocie en permanence et peut varier au sein d'une même société ou d'une même famille selon les époques, comme l'ont montré les périodes de déscolarisation et de rescolarisation des deux dernières décennies. Les stratégies scolaires se construisent ainsi au sein d'un espace-temps déterminé. L'univers scolaire est alors différemment approprié, utilisé, car les différentes sphères (politique, socio-économique, idéologique) influencent les stratégies éducatives de façon concomitante et différentielle. Le choix des enfants à scolariser (filles ou garçons, aînés ou cadets), le nombre d'enfants destinés à la scolarisation, le temps accordé à l'École (durée des études) sont autant d'éléments qui composent et organisent les stratégies scolaires et qui ne se calquent pas nécessairement sur l'organisation du système scolaire ni sur le sens donné à l'instruction par les instances étatiques. L'adhésion ou la non-adhésion aux politiques éducatives impulsées et financées de l'extérieur ne reflètent donc que partiellement la façon dont les populations pensent l'École : c'est bien toute l'ambiguïté de ce processus apparent d'uniformisation.

Les conférences et les aides financières internationales, en tant que véhicules des politiques de mondialisation, permettent la diffusion et l'imposition de 
nouvelles normes scolaires. La dépendance financière de certains pays africains (en particulier des États les plus pauvres) vis-à-vis des bailleurs de fonds s'accroît de telle sorte que les politiques nationales de ces pays sont de plus en plus impulsées et financées de l'extérieur, d'où une certaine uniformisation des réformes scolaires à l'échelle du continent africain. Cependant, le processus de mondialisation est loin de n'être qu'un processus d'uniformisation : la gestation idéologique en cours produit plutôt, par-delà une opposition ou un consensus factuel - faux-semblants dont il convient de décrypter le sens - la diversité des normes, l'éclatement des centres de décision, de nouveaux rapports entre les acteurs.

L'analyse des expressions de la demande scolaire, des représentations sociales de l'École, incite à relativiser le processus d'imposition de normes internationales. Le nouvel ordre scolaire mondial se construit en juxtaposition de dynamiques sociales qui réinterprètent, contournent et travestissent l'idéologie dominante. La tendance à l'uniformisation des systèmes et le triomphe de l'idéologie scolaire se heurtent à la production et à la reproduction de normes sociales et culturelles autonomes. D'un côté, on observe l'élaboration de politiques d'éducation qui répondent au projet universaliste de l'école primaire universelle et du droit à l'éducation, de l'autre, des populations qui, bien que manifestant parfois leur adhésion au projet scolaire, élaborent des représentations du droit à l'éducation qui apparaissent de plus distantes des conceptions occidentales à visée universelle. Le consensus global et apparent des différents acteurs face à l'École s'effrite à la lecture des stratégies familiales d'éducation et des rapports des populations à l'École et les soubassements de ce consensus peuvent apparaître antinomiques. Dans ces conditions, l'École universelle a-t-elle réellement des chances de prendre forme en Afrique ? Cependant, à la dépendance financière et administrative accrue des pays africains répond la dépendance idéologique des institutions internationales et des pays occidentaux, piégés dans leur course à imposer, coûte que coûte, les projets et les valeurs qu'ils considèrent comme univer$\operatorname{sels}^{23}$. D'une certaine façon, les politiques de mondialisation induisent aussi de la réciprocité dans la dépendance...

Institut de recherche pour le développement (IRD), Bondy.

23. En effet, si ces bailleurs de fonds venaient à retirer à la fois leur capacité d'expertise, de gestion et de financement de l'éducation, on observerait aussitôt un recul important de la scolarisation en Afrique. Par ailleurs, ils sont également « piégés » par les modalités d'imposition de leurs réformes scolaires, liant motivations financières - et parfois tolérance dans les pratiques de corruption générées par l'offre (DAHOU 2002) — à la mise en place des réformes. Les rares tentatives opérées pour tenter de «moraliser» ces rapports entre bailleurs de fonds et cadres nationaux se soldent souvent par un échec, comme récemment au Burkina Faso, où les représentations des différentes coopérations ont signé un accord renonçant à l'attribution de per diem lorsque les réunions de travail se tenaient à Ouagadougou, lieu de résidence de ces cadres. Ces coopérations étrangères furent tout aussi nombreuses à signer cet accord qu'à ne pas le respecter, et à s'accuser ensuite mutuellement de ce non-respect. 


\section{BIBLIOGRAPHIE}

Bourdieu, P.

2002 «Pour un savoir engagé », Le Monde diplomatique, février : 3.

BRENNER, L.

2000 Controlling Knowledge. Religion, Power and Schooling in a West African Muslim Society, London, Hurst \& Company.

BROCK-UTNE, B.

1996 «Globalisation of Learning. The Role of the Universities in the South : With a Special Look at Sub-saharan Africa », International Journal of Educational Development, 16 (4) : 335-346.

CARNOY, M.

1999 Mondialisation et réforme de l'éducation : ce que les planificateurs doivent savoir, Paris, UNESCO-IIPE.

Chediel, R. W., Sekwao, N. \& Kirumba, P. L.

2000 Private and Community Schools in Tanzania (Mainland), Paris, UNESCOIIPE.

Cissé, M., Diarra, A., Marchand, J. \& Traoré, S.

2000 Les écoles communautaires au Mali, Paris, UNESCO-IIPE.

COMPAORÉ, M. \& LANGe, M.-F.

2002 Les indicateurs du droit à l'éducation. Collecte de données sur les indicateurs du droit à l'éducation dans la zone de Nomgana, Association pour la promotion de l'éducation non formelle au Burkina (APENF-Burkina Faso), Institut interdisciplinaire d'éthique et des droits de l'Homme et Chaire d'histoire et de politique économiques de l'Université de Fribourg (Suisse), Ouagadougou.

DAHOU, T.

2002 «Déculturaliser la corruption», Les Temps modernes, 620-621 : 289-311.

DÉCLARATION MONDIALE SUR L'ÉDUCATION POUR TOUS

1990 Jomtien.

Diarra, D., Fall, M., Gueye, P. M. \& Marchand, J.

2000 Les écoles communautaires de base au Sénégal, Paris, UNESCO-IIPE.

Esquieu, P. \& PÉANo, S.

1994 L'enseignement privé et spontané dans le système éducatif tchadien, Paris, UNESCO-IIPE.

FORUM MONDIAL SUR L'ÉDUCATION

2000 Synthèse globale. Éducation pour tous. Bilan à l'an 2000, Forum consultatif international sur l'éducation pour tous, Paris, UNESCO. 
Gbogbotchi, K. K. A., Gnossa, K. E., Kpeglo, M. M. \& Marchand, J.

2000 Les écoles d'initiatives locales au Togo, Paris, UNESCO-IIPE.

HALLAK, J.

1994 Au-delà de Jomtien... L'éducation pour tous : grandes espérances ou faux espoirs?, Contribution de l'IIPE, 3, Paris, IIPE-UNESCO.

Henriot-Van Zanten, A.

1988 «Les familles face à l'École : rapports institutionnels et relations sociales », in P. DURNING (dir.), Éducation familiale. Un panorama des recherches internationales, Vigneux, Matrice : 185-207.

INSTITUT INTERNATIONAL DE PLANIFICATION DE L'ÉDUCATION (IIPE)

1987 Les formes traditionnelles d'éducation et la diversification du champ éducatif: le cas des écoles coraniques, rapport d'un séminaire de l'IIPE, Paris, 10-12 décembre 1984, Paris, IIPE.

JODELET, D. (dir.)

1991 [1989] Les représentations sociales, Paris, PUF.

LANGE, M.-F.

1987 «Le refus de l'école : pouvoir d'une société civile bloquée ?», Politique africaine, $27: 74-86$.

1998 L'école au Togo. Processus de scolarisation et institution de l'école en Afrique, Paris, Karthala.

2000 « Naissance de l'école en Afrique subsaharienne », in «Éducations, société », Pour (Revue du Groupe de recherche pour l'éducation et la prospective), $165: 51-59$.

2001 «Dynamiques scolaires contemporaines au Sud» in M.-F. LANGE (dir.), « Des écoles pour le Sud. Stratégies sociales, politiques étatiques et interventions du Nord », Autrepart, $17: 5-12$.

LANGe, M.-F. \& Martin, J.-Y. (dir.)

1995 «Les stratégies éducatives en Afrique subsaharienne», Cahiers des sciences humaines, 31 (3) : 563-737.

Lange, M.-F. \& Diarra, S. O.

1999 «École et démocratie : l'“explosion” scolaire sous la III ${ }^{\mathrm{e}}$ République au Mali », Politique africaine, 76, décembre : 164-172.

Lange, M.-F., Zoungrana, C. \& Yaro, Y.

2002 «Éducation, enfants et sociétés de demain. Exemples africains », Colloque international Enfants d'aujourd'hui. Diversité des contextes, pluralité des parcours, AIDELF, 10-13 décembre, Dakar.

Laval, C. \& Weber, L. (coord.)

2002 Le nouvel ordre éducatif mondial. OMC, Banque Mondiale, OCDE, Commission européenne, Paris, Éditions Nouveaux Regards/Syllepse. 
Le Monde diplomatique

1997 «Un débat Le Monde diplomatique/Financial Times. La mondialisation estelle inévitable? », juin : 14-17.

LES TEMPS MODERNES

2002 «Afriques du monde», août-novembre, 620-621: 46-544.

Nomaye, M.

2001 Les politiques éducatives au Tchad (1960-2000), Paris, L'Harmattan.

OTAYEK, R.

1993 Le radicalisme islamique au sud du Sahara. Da'wa, arabisation et critique de l'Occident, Paris, Karthala-MSHA.

STEWART, F.

1996 «Globalisation and Education », International Journal of Educational Development, 16 (4) : 327-333.

UNESCO

2000 Rapport mondial sur l'éducation 2000. Le droit à l'éducation. Vers l'éducation pour tous, tout au long de la vie, Paris, UNESCO.

VINOKUR, A.

2002 «Mondialisation du capital et reconfiguration des systèmes éducatifs des espaces dominés », Informations et commentaires, 118, janvier-mars.

WORLD EDUCATION FORUM

2000 Statistical Document. Education for All 2000 Assessment, International Consultative Forum on Education for All, Paris, UNESCO.

\section{RÉSUMÉ}

L'évolution récente des systèmes éducatifs africains s'inscrit au sein d'un mouvement impulsé à la fois par des politiques de mondialisation et par des dynamiques sociales promues par les sociétés civiles. Le rôle des conférences et des aides financières internationales, perçues comme véhicules des politiques de mondialisation, est interrogé. L'analyse des expressions de la demande scolaire, des représentations sociales de l'École, de la relation famille-École incite à relativiser le processus d'imposition de normes internationales. Le nouvel ordre scolaire mondial se construit en juxtaposition de dynamiques sociales qui réinterprètent, contournent et travestissent l'idéologie dominante. La tendance à l'uniformisation des systèmes et le triomphe de l'idéologie scolaire se heurtent à la production et à la reproduction de normes sociales et culturelles autonomes.

\section{ABSTRACT}

School and Globalization: Toward a New School Order? - The recent evolution of educational systems in Africa fit into a movement driven by globalization policies 
and social forces. Questions are raised about international conferences and aid, which are perceived as vectors of globalization policies. The analysis of the demand for schooling, of social ideas about school and of family-school relations leads to "relativizing" the process of imposing international norms. The new "world school order" is being built in juxtaposition to social forces that reinterpret, circumvent and disguise the dominant ideology. The tendency to uniformize systems and the triumph of a "school ideology" run up against the production and reproduction of autonomous social and cultural norms.

Mots-clés/Keywords : Burkina Faso, Mali, Sénégal, Tchad, Togo, aide internationale, diversification du champ scolaire, écoles communautaires, éducation, enseignement privé, mondialisation, ordre scolaire mondial, politiques d'éducation, privatisation, stratégies éducatives/Burkina Faso, Mali, Senegal, Chad, Togo, international aid, community schools, education, privatization, private schools, globalization, educational strategies, education policies. 